\title{
Biomimetic Cardiac Tissue Culture Model (CTCM) to Emulate Cardiac Physiology and Pathophysiology ex vivo
}

Jessica Miller

University of Louisville

Moustafa Meki

University of Louisville

Qinghui Ou

University of Louisville

Riham Abouleisa

University of Louisville

Xian-Liang Tang

University of Louisville

Abou Bakr Salama

University of Louisville

Ahmad Gebreil

University of Louisville

Cndy Lin

University of Louisville

Hisham Abdeltawab

University of Louisville

Fahmi Khalifa

University of Louisville

Bradford Hill

University of Louisville

Najah Abi-Gerges

Anabios Corporation

Roberto Bolli

University of Louisville

Ayman El-Baz

University of Louisville

Guruprasad Giridharan

University of Louisville

Tamer Mohamed ( $\nabla$ tamer.mohamed@louisville.edu ) 
University of Louisville

\section{Article}

Keywords:

Posted Date: February 17th, 2022

DOI: https://doi.org/10.21203/rs.3.rs-1322108/v1

License: (9) This work is licensed under a Creative Commons Attribution 4.0 International License. Read Full License

Version of Record: A version of this preprint was published at Communications Biology on September 9th, 2022. See the published version at https://doi.org/10.1038/s42003-022-03919-3. 


\section{Abstract}

Background: There is an urgent need for a reliable in vitro system that can accurately replicate the cardiac physiological environment for drug testing. The limited availability of human heart tissue culture systems has led to inaccurate interpretations of cardiac related drug effects. Our group had previously developed a culture system for pig/human heart slices that enables functional and structural viability for 6 days under electric stimulation and enriched media and can reliably demonstrate the subacute toxic effects of anti-cancer therapeutics. However, by day 10 , the slices undergo cardiomyocyte dedifferentiation and fibrotic remodeling, making them inadequate for long-term drug testing.

Aims: To determine whether including physiological mechanical and humoral cues within the culture system can prolong the viability of the heart tissue in culture.

Methods and Results: We have developed a novel cardiac tissue culture model (CTCM) that can electromechanically stimulate heart slices with physiological preload and afterload during each cardiac cycle. After 12 days in culture, this approach was able to partially improve the viability of heart slices, but not to completely maintain their structural integrity. Therefore, following small molecule screening, we found that incorporation of $100 \mathrm{nM}$ tri-iodo-thyronine (T3) and $1 \mu \mathrm{M}$ dexamethasone (Dex) into our culture media preserved microscopic structure of the slices for 12 days. When combined with T3/Dex treatment, the CTCM system was able to maintain the transcriptional profile, viability, metabolic activity, and structure integrity for 12 days at the same levels as the fresh heart tissue. Furthermore, overstretching the cardiac tissue induced cardiac hypertrophy after 6 days in culture, which provides the first proof of concept for the ability of the CTCM to emulate cardiac pathophysiology.

Conclusions: This manuscript describes a new physiological culture system for heart slices that incorporates physiological mechanical and humoral cues for maintaining viability and functionality of the heart slices. The CTCM has the potential to emulate cardiac physiology and pathophysiology in culture for extended period of time, thereby enabling reliable drug screening.

\section{Introduction}

Prior to clinical studies, there is a need to use reliable in vitro systems that can accurately replicate the human heart's physiological environment. Such systems should model altered mechanical loading, heart rate, and electrophysiological properties. Animal models, which are the prevalently used cardiac physiology screening platform, have limited reliability in mirroring the effects of drugs seen in human hearts. ${ }^{1,2}$. Additionally, using of animal models to create pharmacokinetic profiles of drugs is relatively expensive at the early development stages, due to the sheer quantity of the drugs that are tested ${ }^{3}$. Ultimately, the ideal experimental cardiac tissue culture model (CTCM) is the one that demonstrates high sensitivity and specificity for various therapeutic and pharmacological interventions while accurately replicating the physiology and pathophysiology of the human heart ${ }^{4}$. The lack of such a system has 
limited drug discovery for new heart failure therapeutics 5,6 and resulted in drug induced cardiotoxicity being a major cause of market withdrawal ${ }^{7}$.

In the last decade of the 20th century, eight non-cardiovascular drugs were withdrawn from clinical use because they induce QT interval prolongation that resulted in ventricular arrhythmia and sudden death ${ }^{8}$. Therefore, there is a growing need for reliable preclinical screening strategies to assess cardiovascular efficacy and toxicity. The recent shift towards the use of human induced pluripotent stem cell-derived cardiomyocytes (hiPS-CMs) in drug screening and toxicity testing has provided a partial solution to this issue; however, the immature nature of the hiPS-CMs and loss of the multicellular complexity of the heart tissue are major limitations of this technology ${ }^{9}$. Recent efforts have shown that this limitation can be partially overcome if early-stage hiPS-CMs, soon after the initiation of spontaneous contractions, are used to form cardiac tissue hydrogels and are subjected to a gradual increase in electrical stimulation over time ${ }^{10}$. However, these hiPS-CMs microtissues lack the mature electrophysiological and contractile properties seen in adult human myocardium. Moreover, the human heart tissue is structurally more complicated, being composed of a heterogeneous mixture of various cell types, including endothelial cells, neurons and various types of stromal fibroblasts linked together with a very specific array of extracellular matrix proteins ${ }^{11}$. This heterogeneity of the non-cardiomyocyte cell population ${ }^{12-14}$ in the adult mammalian heart is a major obstacle in modeling heart tissue using individual cell types. These major limitations highlight the importance of developing methods to culture intact myocardial tissue under physiological and pathological conditions ${ }^{10}$.

Cultured thin $(300 \mu \mathrm{m})$ human heart slices are providing a promising model of the intact human myocardium. This technology provides access to a complete 3D multicellular system that is similar to the human heart tissue. However, the use of heart slices has been severely limited by the short period of viability in culture, which does not extend beyond 24 hours using the most robust protocols reported to date ${ }^{15-17}$. This is due to multiple factors including the lack of physiologic mechanical loading, the airliquid interface, and the use of a simple culture medium that does not support the demands of the cardiac tissue. Our lab has previously used electrical stimulation and an optimized culture medium to keep cardiac tissue slices viable for up to 6 days ${ }^{18,19}$. This culture system has the potential to become a powerful predictive in situ human model for efficacy and cardiotoxicity testing to close the gap between preclinical and clinical testing results. Multiple studies have demonstrated the ability to culture heart slices for extended period of time, at the functional and transcriptional levels ${ }^{20-22}$. In the current manuscript, we describe a novel CTCM that fully recapitulates the cardiac physiology and pathophysiology during the cardiac cycle. This СTCM has the potential to advance the accuracy of preclinical drug prediction to previously unattainable levels by providing a medium-throughput, cost-effective human heart system that faithfully mimics mammalian cardiac physiology/pathophysiology for preclinical drug testing.

\section{Methods}




\section{Heart tissue collection from pigs}

To collect hearts from the 13 pigs used in the current study, all animal procedures were in accordance with the institutional guidelines and approved by the University of Louisville Institutional Animal Care and Use Committee. The aortic arch was clamped and hearts were perfused with $1 \mathrm{~L}$ of sterile cardioplegia solution $\left(110 \mathrm{mM} \mathrm{NaCl}, 1.2 \mathrm{mM} \mathrm{CaCl}_{2}, 16 \mathrm{mM} \mathrm{KCl}, 16 \mathrm{mM} \mathrm{MgCl}_{2}, 10 \mathrm{mM} \mathrm{NaHCO}_{3}, 5\right.$ units/ml heparin, $\mathrm{pH}$ to 7.4); the hearts were preserved on ice-cold cardioplegic solution until transported to the lab on wet ice.

\section{CTCM device fabrication}

The CTCM devices were designed in SolidWorks Computer Aided Design (CAD) software. The culture chamber, separating plate, and air chamber were manufactured using CNC (computer numerical control) machining out of clear acrylic plastic. The $7 \mathrm{~mm}$ support rings were center-lathed from high density polyethylene plastic (HDPE) and fitted with an 0-ring groove to accommodate silicone O-rings for sealing the media below. A thin silicone membrane separates the culture chamber from the separating plate. The silicone membrane was laser-cut out of 0.02 " silicone sheet, durometer 35A. The bottom and top silicone gaskets were laser-cut from 1/16" silicone sheet, durometer 50A. 316 L stainless steel screws and wing nuts were used to hold the device together to create an air-tight seal.

A custom PCB was designed to integrate with the C-PACE-EM system. Swiss machine headers outlets on the PCB are each connected to a graphite electrode via silver plated copper wires and 0-60 bronze screws that are threaded into the electrodes. The PCB fits into a 3D printed device cover.

The CTCM device is controlled by a programmable pneumatic driver (PPD) that can induce a controlled cyclic pressure similar to the cardiac cycle. As the pressure within the air chamber increases, the flexible silicone membrane will distend upward displacing the culture medium under the tissue slices. The tissue slices will then be stretched by this fluid displacement, mimicking cardiac preload. At the peak of this cardiac preload, electrical stimulation is applied through the graphite electrodes and the air chamber pressure is decreased allowing for the tissue slices to contract. Within the airline, there is a hemostatic valve with a pressure probe sensor that detects the pressure of the air system. The pressure detected by the pressure sensor is fed into a data acquisition (DAQ) device that is connected to a laptop. This allows for the continuous monitoring of the pressures within the air chamber. When the maximum pressure is reached, the DAQ is told to send out a signal to the C-PACE-EM system inducing a $2 \mathrm{~ms}$ biphasic electrical voltage signal set at $4 \mathrm{~V}$.

\section{Heart slice culture}

Heart slices were obtained, and 6-well culture conditions were performed as previously described in ${ }^{18,19}$. For TD conditions, fresh T3 and Dex were added at $100 \mathrm{nM}$ and $1 \mu \mathrm{M}$ concentrations, respectively at each media change. The culture media was changed 3 times a day.

For CTCM culture, the tissue slices were positioned on a custom 3D-printed apparatus within a petri dish containing modified Tyrode's solution. The apparatus is designed to oversize the heart slice by $25 \%$ of the 
support ring area. This is done to ensure that the heart slice is not over stretched once it has been transferred from the Tyrode's solution to culture media and during the diastolic phase. Using histoacryl glue, the $300 \mu \mathrm{m}$ thick slices were fixed to 7-mm diameter support rings. Once the tissue slice was attached to the support ring, the excess tissue slice was trimmed off and the attached tissue slice is placed back into the Tyrode's solution bath until enough slices were prepared for one device. Once 6 tissue slices were attached to their support rings, the CTCM device was assembled. The CTCM culture chamber was prefilled with $21 \mathrm{~mL}$ of culture media (Medium 199, 1x ITS supplement, 10\% FBS, $5 \mathrm{ng} / \mathrm{mL}$ VEGF, $10 \mathrm{ng} / \mathrm{mL}$ FGF-basic, and 2X Antibiotic-Antimycotic). The tissue slices were transferred into the culture chamber and all air bubbles were carefully removed with a pipette. Then the tissue slice was guided over to a well and gently pressed into place. Lastly, the electrode cover was placed onto the device and the device was transferred to an incubator. The CTCM was then connected to the air tubes and the CPACE-EM system. The pneumatic driver was then switched on and the air flow valve is opened to the CTCM device. The C-PACE-EM system was set to provide $4 \mathrm{~V}$ at $1.2 \mathrm{~Hz}$ at $2 \mathrm{~ms}$ biphasic stimulation. The culture medium was changed twice per day and the electrodes were replaced once a day to avoid graphite buildup from the electrodes. If necessary, the tissue slices can be removed from their culture wells to displace any air bubbles that may be trapped below. For MT conditions, the T3+Dex treatment was added fresh with every media change at $100 \mathrm{nM}$ T3 and $1 \mu \mathrm{M}$ Dex.

\section{Heart slice mechanical stretches assessment using MUSCLEMOTION}

To obtain a stretch trace of the heart slices, a custom camera system was developed. A DSLR camera (Cannon Rebel T7i, Cannon, Tokyo, Japan) was used with a Navitar Zoom 7000 18-108mm Macro Lens, (Navitar, San Francisco, CA). The camera was placed at a $51^{\circ}$ angle and videos were recorded at $30 \mathrm{fps}$. Firstly, an open-source software (MUSCLEMOTION, ${ }^{23}$ ) was used with Image-J to quantify the movement of the heart slices. A mask was created using MATLAB (MathWorks, Natick, MA, USA) to define the region of interest which is the beating heart slice to avoid any noise. The manually segmented mask was applied to all images in the frame sequence and then fed to the MUSCLEMOTION plugin. MUSCLE motion uses the average pixel intensity within each frame to quantify its movement relative to a reference frame. The data was recorded, filtered, and used to quantify the cycle time and assess the tissue stretches during the cardiac cycle. Post processing of recorded videos was performed using a zero phase first order digital filter. To quantify tissue stretch (peak to peak), peak analysis was performed to distinguish the peaks and valleys of the recorded signal. Additionally, detrending was performed to remove signal drift using a 6th order polynomial. Software code was developed in MATLAB to detect overall tissue movement, cycle time, relaxation time, and contraction time (Supplementary code).

\section{Heart slice fixation and mounting}

Heart slices were fixed in $4 \%$ paraformaldehyde for 48 hours. Fixed tissue underwent dehydration in $10 \%$ then $20 \%$ sucrose for 1 hour followed by $30 \%$ sucrose overnight. The slices were then embedded in optimal cutting temperature compound (OCT compound) and gradually frozen in isopentane/dry ice 
bath. OCT embedded blocks were stored at $-80^{\circ} \mathrm{C}$ until sectioning. Slides were prepared at $8 \mu \mathrm{m}$ thick sections.

\section{Immunostaining}

To remove the OCT from the heart slices, the slides were heated on a heat block for 5 minutes at $95^{\circ} \mathrm{C} .1$ $\mathrm{mL}$ of PBS was added to each slide and incubated at room temperature for 30 minutes. Sections were then permeabilized by incubating for 15 minutes with $0.1 \%$ Triton-X in PBS at room temperature. To prevent non-specific antibody binding to the samples, $1 \mathrm{~mL}$ of $3 \%$ BSA solution was added to the slides and incubated for 1 hour at room temperature. The BSA was then discarded, and the slides were washed with PBS. Using a wax pen, each sample was marked off. The primary antibodies (1:200 dilution in 1\% BSA) (Connexin 43 (Abcam; \#AB11370), and Troponin-T (Thermo Scientific; \#MA5-12960), were added to the section for 90 minutes followed by the secondary antibodies (1:200 dilution in 1\% BSA) Anti-mouse Alexa Fluor 488 (Thermo Scientific; \#A16079), Anti-rabbit Alexa Fluor 594 (Thermo Scientific; \#T6391) for another 90 minutes separated by 3 washes with PBS. To distinguish the target staining from background, we used a secondary antibody only as a control. Finally, the DAPI nuclear stain was added, and the slides were mounted in vectashield (Vector Laboratories) and sealed with nail polish. Immunofluorescence imaging was performed using a Cytation 1 high content imager (20X lens magnification) and a Keyence microscope using 40X lens magnification.

For WGA staining, WGA-Alexa Fluor 555 (Thermo Scientific; \#W32464) was used at $5 \mu \mathrm{g} / \mathrm{mL}$ in PBS and was applied to fixed sections for 30 minutes at room temperature. The slides were then washed with PBS and Sudan Black was added to each slide and incubated for 30 minutes. The slides were then washed with PBS and vectashield mounting media was added. Slides were imaged on a Keyence microscope using 40X lens magnification.

\section{Masson's Trichrome stain}

OCT was removed from the samples as described above. Once the OCT was removed, the slides were submerged in Bouin's solution overnight. The slides were then rinsed with distilled water for 1 hour, followed by placing the slides in in Biebrich Scarlet-acid Fuchsin solution for 10 minutes. The slides were then washed with distilled water and placed into in $5 \%$ phosphomolybdic / $5 \%$ phosphotungstic acid solution for 10 minutes. Without rinsing, the slides were directly transferred into aniline blue solution for 15 minutes. Then the slides were rinsed again with distilled water and placed in $1 \%$ acetic acid solution for 2 minutes. The slides were dried in 200 proof ethyl alcohol and subsequently transferred into xylene. Stained slides were imaged using a Keyence microscope with 10X lens magnification. The percentage area of fibrosis was quantified using the Keyence Analyzer software.

\section{MTT assay}

CyQUANT ${ }^{\text {TM }}$ MTT Cell Viability Assay (Invitrogen, Carlsbad, CA), catalogue \# V13154 was used according to the manufacturer's protocol with some modifications. A $6 \mathrm{~mm}$ surgical punch was used to ensure similar tissue size when performing the MTT assay. The tissues were each placed in a well of 12 well 
plate containing MTT substrate according to the manufacturer's protocol. The slices were incubated for 3 hours at $37^{\circ} \mathrm{C}$ and viable tissue metabolized the MTT substrate creating a purple color formazan compound. To extract the purple formazan from the heart slices, the MTT solution was replaced with 1 $\mathrm{ml}$ of DMSO and incubated it at $37^{\circ} \mathrm{C}$ for 15 minutes. Samples were diluted to $1: 10$ in DMSO within a clear bottom 96 well plate and the intensity of the purple color was measured using a Cytation plate reader (BioTek) at 570nm. The readings were normalized to the weight of each heart slice.

\section{Tritiated glucose utilization assay}

The media of heart slices was changed with a culture medium containing $1 \mu \mathrm{Ci} / \mathrm{ml}[5-3 \mathrm{H}]$-glucose (Moravek Biochemicals, Brea, CA, USA) for glucose utilization assay, as described previously 24,25. Following incubation for $4 \mathrm{~h}, 100 \mu \mathrm{l}$ of media was added to a cap-less microcentrifuge tube containing $100 \mu \mathrm{l}$ of $0.2 \mathrm{~N} \mathrm{HCl}$. Then the tube was placed in a scintillation vial containing $500 \mu \mathrm{l}$ of $\mathrm{dH}_{2} \mathrm{O}$ to allow for evaporation diffusion of $\left[{ }^{3} \mathrm{H}\right]_{2} \mathrm{O}$ at $37^{\circ} \mathrm{C}$ for 72 hours. Then, the microcentrifuge tubes were removed from the scintillation vials, and $10 \mathrm{ml}$ of scintillation fluid were added. Scintillation counting was performed using a Tri-Carb 2900TR Liquid Scintillation Analyzer (Packard Bioscience Company, Meriden, CT, USA). Glucose utilization was then calculated, with considerations for the specific activity of $\left[5^{-}{ }^{3} \mathrm{H}\right]$-glucose, incomplete equilibration and background, dilution of $\left[5-{ }^{3} \mathrm{H}\right]$-to unlabeled-glucose, and scintillation counter efficiency. Data were normalized to the weight of the heart slices.

\section{RNAseq}

RNA was isolated from the heart slices by using the Qiagen miRNeasy Micro Kit, \#210874, following the manufacturer's protocol after homogenization of tissue in Trizol. RNAseq library preparation, sequencing, and data analysis were performed as described previously ${ }^{19}$.

\section{NT- ProBNP}

NT-ProBNP release in the culture media was assessed using a NT-ProBNP kit (pigs) (Cat\#MBS2086979, MyBiosource) according to the manufacturer protocol. Briefly, $250 \mu \mathrm{L}$ of each sample and standard were added each well in duplicate. Immediately following the addition of the samples, $50 \mu \mathrm{L}$ of Detection Reagent A was added per well. The plate was shook gently and sealed with a Plate Sealer. The plate was then incubated for 1 hour at $37^{\circ} \mathrm{C}$. Then, the solution was aspirated, and the wells were washed with 350 $\mu \mathrm{L}$ of $1 \mathrm{X}$ Wash Solution 4 times, letting the Wash Solution incubate for 1-2 minutes each time. Next, 100 $\mu \mathrm{L}$ of Detection Reagent B was added per cell and sealed with a Plate sealer. The plate was gently shook and incubated at $37^{\circ} \mathrm{C}$ for 30 minutes. The solution was aspirated, and the wells were washed with 350 $\mu \mathrm{L}$ of $1 \mathrm{X}$ Wash Solution 5 times. $90 \mu \mathrm{L}$ of Substrate Solution was added to each well and the plate was sealed. The plate was incubated for $10-20$ minutes at $37^{\circ} \mathrm{C}$. $50 \mu \mathrm{L}$ of Stop Solution was added per well. The plate was immediately measured using a Cytation plate reader (BioTek) set at $450 \mathrm{~nm}$.

\section{Statistical Analysis}


GraphPad Prism software (San Diego, CA) was used to perform all statistical analysis. For all statistics pvalues were considered significant at values less than 0.05 . Two-tailed, Student's t-tests were performed for data with only 2 group comparisons. One-way ANOVA was used to determine significance between multiple groups. To account for multiple comparisons when performing post hoc tests, the Tukey correction was applied.

\section{Results}

CTCM maintains heart tissue physiological stretch for 12 days in culture

Hemodynamic mechanical cues play a critical role in preserving the functionality of cardiomyocytes (CM) in vitro ${ }^{26-28}$. In the current manuscript, we developed a CTCM (Fig. 1a) that can emulate the adult cardiac milieu by inducing simultaneous electrical and mechanical stimulation at the physiological frequency $(1.2 \mathrm{~Hz}, 72$ beats per minute). To avoid over stretching the tissue during the diastolic phase the tissues were oversized by $25 \%$ using a 3D-printed apparatus (Fig. $1 \mathrm{~b}$ ). To fully reproduce the cardiac cycle, electrical stimulation induced by a C-PACE system was synchronized to initiate at $100 \mathrm{~ms}$ prior to the systolic phase using a data acquisition system (DAQ). The tissue culture system uses a programmable pneumatic driver (LB engineering, Germany) to cyclically distend a flexible silicone membrane inducing the stretch of the heart slices in the chambers above. The system is connected to an external air line with a pressure probe allowing fine pressure tuning $( \pm 1 \mathrm{mmHg})$ and timing $( \pm 1 \mathrm{~ms})$ (Fig. 1C). Using one pneumatic driver we can operate 4 CTCM devices, each accommodating 6 tissue slices (Fig. 1d). Within the CTCM, the air pressures in the air chambers are translated into synchronized pressures in the fluid chamber and produce physiological stretch of the heart slices (Fig. 2a, supplementary movie 1). Assessment of the tissue stretch at $80 \mathrm{mmHg}$ resulted in $25 \%$ stretch of the tissue slice (Fig. 2b). This percent stretch has been shown to correspond with a physiological sarcomere length, 2.2-2.3 um, for normal contractility of heart slices ${ }^{20,22,29}$. Tissue movements are assessed using a custom camera setup (Supplementary Fig. 1). The tissue movement amplitude and speed rate (Fig. 2c-d) are consistent with the stretching during the cardiac cycle and the timing during systole and diastole (Fig. 2b). The heart tissue stretch and speed during contraction and relaxation remained consistent over the 12 days in culture (Fig. 2e).

\section{Heart slices cultured in CTCM maintain full viability but not structural integrity over 12 days.}

In our previous static biomimetic heart slice culture system ${ }^{18,19}$, we were able to maintain the viability, functionality, and structural integrity of heart slices for 6 days with the application of electrical stimulation and optimized composition of the culture medium. However, after day 10 and 12, there was a sharp decline of these parameters. We will refer to slices cultured in our previous static biomimetic culture system ${ }^{18,19}$ as control condition (Ctrl) and we will refer to the slices cultured under synchronized mechanical and electrical stimulation (CTCM) using our previously optimized culture media as MC condition. Interestingly, by introducing physiological mechanical stimulation using the CTCM, the viability of 12-day heart slices was maintained similar to fresh heart slices in MC condition but not in the Ctrl 
condition as shown by MTT assay (Fig. 3a). This indicates that the mechanical stimulation and simulation of the cardiac cycle can maintain the viability of tissue slices for twice the time reported in our previous static culture system. However, assessment of the structural integrity of the tissue slices as assessed by immunolabeling for cardiac Troponin-T and Connexin 43 demonstrated that connexin 43 expression of day $12 \mathrm{MC}$ tissue was significantly higher than the same day control, but the uniform expression of connexin43 and z-disc formation were not fully maintained. Using an artificial intelligence (Al) based framework to quantify tissue structural integrity ${ }^{30}, \mathrm{MC}$ tissue showed an improved structural similarity to day 0 compared to the static control slices. Furthermore, Masson's trichrome stain showed a significantly lower percent area of fibrosis with the MC condition compared to the control condition at day 12 of culture. While the CTCM did improve the viability of day 12 heart tissue slices to levels similar to fresh heart tissue, it did not significantly improve the structural integrity of the heart slices.

\section{Small molecule screening to improve the heart tissue viability and structural integrity in CTCM}

We hypothesized that by incorporating small molecules into the culture media cardiomyocyte integrity could be improved and the development of fibrosis during the CTCM culture could be reduced. Therefore, we performed a small molecule screening using our static control culture ${ }^{18,19}$ because of the lower number of confounding factors associated with it. Dexamethasone (Dex), tri-iodothyronine (T3), and SB431542 (SB) were selected for this screening. These small molecules have been previously used in hiPSC-CM cultures to induce cardiomyocyte maturation by improving sarcomere length, t-tubules, and conduction velocity 31,32 . Additionally, both Dex, a glucocorticoid, and SB are known to suppress inflammation ${ }^{33,34}$. Therefore, we tested whether incorporating one or a combination of these small molecules would improve the structural integrity of the heart slices. For the initial screening the dosage of each compound was selected based on the concentration typically used in cell culture models $(1 \mu \mathrm{M}$ Dex ${ }^{31}, 100 \mathrm{nM} \mathrm{T3}{ }^{31}$, and $2.5 \mu \mathrm{M} \mathrm{SB}^{35}$ ). Following 12 days in culture, the combination of T3 and Dex resulted in the best cardiomyocyte structural integrity and the lowest fibrotic remodeling (Supplementary Fig. 2-3). In addition, using either half or double of these concentrations of T3 and Dex had detrimental effects compared to the normal concentrations (Supplementary Fig. 4).

\section{The combination of T3 and Dex with the CTCM fully maintained pig heart slices similar to fresh heart slices for 12 days.}

Following the initial screening, we performed head-to-head comparisons of 4 culture conditions; Ctrl: heart slices cultured in our previously described static culture with our optimized culture media ${ }^{18,19}$; TD: Ctrl with T3 and Dex added to the culture media; MC: heart slices cultured in CTCM using our previously optimized culture media; and MT: CTCM with T3 and Dex added to the culture media. After 12 days in culture, the viability of MC and MT tissues was maintained similar to fresh tissue as assessed by the MTT assay. Interestingly, the addition of T3 and Dex to the transwell culture (TD) did not significantly improve viability compared to Ctrl condition, implying the vital role of mechanical stimulation in maintaining heart slice viability. 
Metabolic shift from fatty acid oxidation to glycolysis is a hallmark for cardiomyocyte dedifferentiation. Immature cardiomyocytes primarily utilize glucose for ATP production and have underdeveloped mitochondria with few cristae ${ }^{636}$. Glucose utilization assay demonstrated that under MC and MT conditions, glucose utilization was similar to day 0 tissue. However, Ctrl samples showed a significant increase in glucose utilization compared to fresh tissue. This indicates that the combination of CTCM and T3+Dex improved the viability and metabolic activities in heart slices cultured for 12 days.

To analyze the overall impact of the CTCM and T3+Dex on the global transcriptional landscape of the heart slice tissue, we performed RNAseq on heart slices from all 4 different culture conditions (Supplementary source data). Interestingly, MT slices showed high transcriptional similarity to the fresh heart tissue with only 16 out of 13,642 genes differentially expressed. However, as we demonstrated before ${ }^{19}$, the Ctrl slices showed 1,229 differentially expressed gene after 10-12 days in culture (Fig. 5D). Interestingly, the Ctrl slices showed downregulation of the cardiac genes and cell cycle genes and an upregulation of inflammatory gene programs. These data indicate that the dedifferentiation that normally occurs following long-term culture, was completely attenuated under the MT condition (Supplementary Fig. 5). A closer examination of the sarcomeric genes revealed that only the MT conditions that preserved the sarcomeric genes from downregulation seen in Ctrl, TD, and MC conditions (Fig. 5E). These data indicate that with the combination of mechanical and humoral stimulation (T3+Dex), the transcriptome of heart slices could be maintained similar to fresh heart slices for 12 days in culture.

These transcriptional findings were confirmed by the fact that the structural integrity of the cardiomyocytes in heart slices was best preserved for 12 days in the MT condition as shown by the intact and localized gap junction protein, connexin 43 (Fig. 5A). Furthermore, fibrosis in heart slices in MT condition was significantly reduced compared to Ctrl and was similar to the fresh heart slices (Fig. 5B). These data indicate that the combination of mechanical stimulation and T3+Dex treatment was able to effectively preserve heart slice cardiac structure for extended time in culture.

\section{Inducing cardiac hypertrophy through overstretching the tissue in the СТСM}

Lastly, the ability of the CTCM to model overload cardiac hypertrophy was assessed by increasing the cardiac tissue stretch amplitude. In the СТCM, the peak pressure in the air chamber was increased from $80 \mathrm{mmHg}$ (normal stretch) to $140 \mathrm{mmHg}$ (Fig. 6a). This corresponds to an increase in the preload stretch of $32 \%$ (Fig. 6b), which has been previously shown to be the appropriate percent stretch necessary for a heart slice to achieve a sarcomere length similar to that seen in hypertrophy $20,22,29$. The heart tissue stretch and speed during contraction and relaxation remained consistent over the 6 days of culture (Fig. 6c). Heart slices tissue from the MT conditions were either subjected to normal preload stretch (MT (Norm)) or overload conditions (MT (OL)) for 6 days. As early as 4 days in culture, the hypertrophic biomarker, NT-proBNP, was significantly increased in the culture media in MT (OL) conditions compared to the MT (Norm) conditions (Fig. 7a). Furthermore, following 6 days in culture, the cell size in MT (OL) (Fig. 7b) was significantly increased compared to MT (Norm) heart slices. These results show the 
progressive development of pathological remodeling following overload and provide a proof of concept that the CTCM device can be used as a platform to induce overload cardiac hypertrophy.

\section{Discussion}

Translational cardiovascular research requires cellular models capable of faithfully replicating the cardiac milieu. In this study, a CTCM that can stimulate ultra-thin heart slices was developed and characterized. The CTCM system encompasses physiological synchronized electromechanical stimulation coupled with humoral enrichment with T3 and Dex. By subjecting pig heart slices to these factors, the viability, structural integrity, metabolic activity, and transcriptional expression were all maintained similar to fresh heart tissue for 12 days in culture. Furthermore, overstretching the heart tissue incites overload-induced cardiac hypertrophy. Overall, these results confirm the critical role of physiological culture conditions in the maintenance of a normal cardiac phenotype and provide a new platform for drug screening.

Multiple factors contribute to the optimal environment for cardiomyocyte function and survival. The most obvious of these factors are related to 1) cell-to-cell interactions, 2) electromechanical stimulation, 3) humoral factors, and 4) metabolic substrates. Physiological cell-to-cell interaction requires the presence of intricate 3-dimensional multi-cell-type networks supported by an extra cellular matrix. This complex interaction of cells is difficult to recreate in vitro through the co-culture of individual cell types, but can be easily accomplished by utilizing the organotypic nature of the heart slices.

Mechanical loading and electrical stimulation of cardiomyocytes are essential for preserving the cardiac phenotype ${ }^{37-39}$. While mechanical stimulation has been used extensively in hiPSC-CM conditioning and maturation, few studies have attempted mechanical loading of heart slices in culture ${ }^{20}$. Two elegant studies, ${ }^{20}$ and ${ }^{21}$, have shown the positive influence of mechanical loading on the cardiac phenotype during culture. However, these studies did not use the dynamic 3D physiological mechanical loading of the cardiac cycle, since heart slices were either loaded with isometric stretch forces ${ }^{20}$ or linear auxotonic loading ${ }^{21}$. These methods of stretching the tissue resulted in the downregulation of multiple cardiac genes or the overexpression of genes related to pathological stretch response. Notably, Pitoulis et al. ${ }^{22}$ developed a dynamic heart slice culture bath to recreate the cardiac cycle using force transducer feedback and a stretcher actuator. While this system allowed for the cardiac cycle to be more accurately simulated in vitro, the complexity and low throughput of the method limits the application the system. The CTCM described here, can provide 3D electromechanical stimulation that mimics the native cardiac cycle in terms of cycle timing and loading (25\% preload stretch, $40 \%$ systole, $60 \%$ diastole, and 72 beats per minutes). Further support for the concept that the mechanical stimulation provided by the CTCM can accurately model the hemodynamics of the heart is evinced from the fact that the pathological state of overload-induced cardiac hypertrophy was reproduced.

Humoral factors play an essential role in regulating adult cardiac phenotype. This has been emphasized in hiPS-CM studies that incorporate T3 and Dex in the culture medium to promote the maturation of the 
cells. T3 can influence transport of amino acids, sugars, and calcium across the cell membrane ${ }^{40}$. Additionally, T3 promotes the expression of MHC- $a$ and downregulation of MHC- $\beta$, promoting fast-twitch myofibrils seen in mature cardiomyocytes vs. slow-twitch myofibrils seen in fetal $\mathrm{CMs}^{41}$. The lack of T3 in hypothyroid patients can lead to the loss of myofibrillar striation and reduced rate of tension development ${ }^{41}$. Dex acts on the glucocorticoid receptor and has been shown to increase cardiac contractility in ex vivo perfused hearts ${ }^{42}$, an improvement is thought to be related to effects on store operated calcium entry (SOCE) ${ }^{43,44}$. Additionally, when Dex binds to its receptor, it induces a wide range of intracellular responses that suppress immune function and inflammation ${ }^{34}$.

Our results demonstrate that physiological mechanical stimulation (MC) improved overall culture performance compared to Ctrl, but cannot, in itself, maintain viability, structural integrity, and cardiac expression for 12 days in culture. The incorporation of T3 and Dex treatment in the CTCM culture (MT) resulted in improved viability compared to Ctrl and maintained transcriptional profile, structural integrity, and metabolic activity similar to fresh heart tissue for 12 days. Furthermore, a model of overload-induced cardiac hypertrophy modeling using the CTCM was achieved by controlling the extent of the tissue stretch, illustrating the versatility of the CTCM system. It should be noted that the CTCM parameters can be modulated to simulate many conditions such as tachycardia, bradycardia, and mechanical circulatory support (mechanically unloaded heart) by changing the pressure/electrical amplitudes and frequencies. This allows for the system to be a medium-throughput platform for drug testing. The ability of the CTCM to model overload-induced cardiac hypertrophy opens the avenue for this system to be utilized in personalized therapy testing. The present study demonstrates that both hemodynamic loading and humoral stimulation are essential factors to incorporate in cardiac tissue slice cultures.

\section{Limitations Of The Study}

While the data shown here indicate that the CTCM is a very promising platform for modeling the intact myocardium, there are a few limitations to this novel culture method. The major limitation of the CTCM culture is that it applies continuous dynamic mechanical loading to the slices, which precludes the ability to actively monitor the heart slice contraction during each cycle. Additionally, due to the small size of the heart slices $(7 \mathrm{~mm})$, there is a limited ability to perform contractile function assessment outside the culture system using traditional force transducers. This limitation may be overcome by implementing new methods to optically monitor the functionality of the heart slices within the culture, such as strain analysis and optical mapping using calcium and voltage sensitive dyes.

\section{Declarations}

\section{Funding Sources}

T.M.A.M. is supported by NIH grants R01HL147921 and P30GM127607 and American Heart Association grant 16SDG29950012. The authors also acknowledge NIH grants F32HL149140 (R.R.E.A.), P30GM127607 (B.G.H.), R01HL130174 (B.G.H.), R01HL147844 (B.G.H.), R01ES028268 (B.G.H.), 
P01HL78825 (R.B., B.G.H.), UM1HL113530 (R.B.), and 2U54HL120163. We also acknowledge the United States of America Department of Defense for the grant W81XWH-20-1-0419 (T.M.A.M.).

\section{Author contributions}

J.M.M and M.H.M.: experimental design, collection and analysis of molecular and cellular data, manuscript writing, and final approval of the manuscript; Q.O.: heart cutting, staining, and imaging; X-L.T., R.B.: Pig heart collection and manuscript writing, R.R.E.A and B.G.H.: Metabolic analysis; A.S., C.L. and A.G.: immunostaining and trichrome staining and analysis; H.A., F.K., N.A. and A.S.E: Artificial intelligence analysis for heart slice tissue integrity; G.G.: Engineering design and supervision of the CTCM and mechanical stimulation; T.M.A.M.: conception and design of the overall work, and provided funding. All authors have contributed to the manuscript writing and final approval of the manuscript.

\section{Conflict of Interest Disclosures}

T.M.A.M.: Holds equities at Tenaya Therapeutics. G.G consults for NuPulseCV. All other authors, declare no conflicting interest.

\section{References}

1. Salama, G., Bett, G.C.: Sex differences in the mechanisms underlying long QT syndrome. Am. J. Physiol. Heart Circ. Physiol. 307, H640-H648 (2014). doi:10.1152/ajpheart.00864.2013

2. Liu, W., et al.: MR tagging demonstrates quantitative differences in regional ventricular wall motion in mice, rats, and men. Am. J. Physiol. Heart Circ. Physiol. 291, H2515-H2521 (2006). doi:10.1152/ajpheart.01016.2005

3. Guth, B.D., et al.: Considerations for an In Vitro, Cell-Based Testing Platform for Detection of Adverse Drug-Induced Inotropic Effects in Early Drug Development. Part 1: General Considerations for Development of Novel Testing Platforms. Front. Pharmacol. 10, 884 (2019). doi:10.3389/fphar.2019.00884

4. Wang, D., Patel, C., Cui, C., Yan, G.X.: Preclinical assessment of drug-induced proarrhythmias: role of the arterially perfused rabbit left ventricular wedge preparation. Pharmacol. Ther. 119, 141-151 (2008). doi:10.1016/j.pharmthera.2008.02.009

5. Correale, M., et al.: New Targets in Heart Failure Drug Therapy. Front. Cardiovasc. Med. 8, 665797 (2021). doi:10.3389/fcvm.2021.665797

6. Meki, M.H., Miller, J.M., Mohamed, T.M.: A. Heart Slices to Model Cardiac Physiology. Front. Pharmacol. 12, 617922 (2021). doi:10.3389/fphar.2021.617922

7. Onakpoya, I.J., Heneghan, C.J., Aronson, J.K.: Post-marketing withdrawal of 462 medicinal products because of adverse drug reactions: a systematic review of the world literature. BMC Med. 14, 10 (2016). doi:10.1186/s12916-016-0553-2 
8. Fermini, B., Fossa, A.A.: The impact of drug-induced QT interval prolongation on drug discovery and development. Nature reviews Drug Discovery 2, 439-447, doi:10.1038/nrd1108 nrd1108 [pii]: (2003)

9. Robertson, C., Tran, D.D., George, S.C.: Concise review: maturation phases of human pluripotent stem cell-derived cardiomyocytes. Stem Cells. 31, 829-837 (2013). doi:10.1002/stem.1331

10. Ronaldson-Bouchard, K., et al.: Advanced maturation of human cardiac tissue grown from pluripotent stem cells. Nature. 556, 239-243 (2018). doi:10.1038/s41586-018-0016-3

11. Pinto, A.R., et al.: Revisiting Cardiac Cellular Composition. Circul. Res. 118, 400-409 (2016). doi:10.1161/CIRCRESAHA.115.307778

12. Fu, X., et al.: Specialized fibroblast differentiated states underlie scar formation in the infarcted mouse heart. J. Clin. Investigations. 128, 2127-2143 (2018). doi:10.1172/JCI98215

13. Kanisicak, O., et al.: Genetic lineage tracing defines myofibroblast origin and function in the injured heart. Nat. Commun. 7, 12260 (2016). doi:10.1038/ncomms 12260

14. Kretzschmar, K., et al.: Profiling proliferative cells and their progeny in damaged murine hearts. Proc. Natl. Acad. Sci. U.S.A. 115, E12245-E12254 (2018). doi:10.1073/pnas.1805829115

15. Kang, C., et al.: Human Organotypic Cultured Cardiac Slices: New Platform For High Throughput Preclinical Human Trials. Sci. Rep. 6, 28798 (2016). doi:10.1038/srep28798

16. Perbellini, F., et al.: Investigation of cardiac fibroblasts using myocardial slices. Cardiovasc. Res. 114, 77-89 (2018). doi:10.1093/cvr/cvx152

17. Watson, S.A., et al.: Preparation of viable adult ventricular myocardial slices from large and small mammals. Nat. Protoc. 12, 2623-2639 (2017). doi:10.1038/nprot.2017.139

18. Ou, Q., Tang, A.R., Juhardeen, X.L., Meki, H.R., Miller, M.H., Giridharan, J.M., El-Baz, G., Bolli, A., Mohamed, R.: TMA Slicing and Culturing Pig Hearts under Physiological Conditions. J. Vis. Exp., doi:10.3791/60913 (2020)

19. Ou, Q., et al.: Physiological Biomimetic Culture System for Pig and Human Heart Slices. Circul. Res. 125, 628-642 (2019). doi:10.1161/CIRCRESAHA.119.314996

20. Watson, S.A., et al.: Biomimetic electromechanical stimulation to maintain adult myocardial slices in vitro. Nat. Commun. 10, 2168 (2019). doi:10.1038/s41467-019-10175-3

21. Fischer, C., et al.: Long-term functional and structural preservation of precision-cut human myocardium under continuous electromechanical stimulation in vitro. Nat. Commun. 10, 117 (2019). doi:10.1038/s41467-018-08003-1

22. Pitoulis, F.G., et al.: Remodelling of adult cardiac tissue subjected to physiological and pathological mechanical load in vitro. Cardiovasc. Res., doi:10.1093/cvr/cvab084 (2021)

23. Sala, L., et al.: MUSCLEMOTION: A Versatile Open Software Tool to Quantify Cardiomyocyte and Cardiac Muscle Contraction In Vitro and In Vivo. Circ. Res. 122, e5-e16 (2018). doi:10.1161/CIRCRESAHA.117.312067

24. Gibb, A.A., et al.: Integration of flux measurements to resolve changes in anabolic and catabolic metabolism in cardiac myocytes. Biochem. J. 474, 2785-2801 (2017). doi:10.1042/BCJ20170474 
25. Abouleisa, R.R.E., et al.: Cell cycle induction in human cardiomyocytes is dependent on biosynthetic pathway activation. Redox Biol. 46, 102094 (2021). doi:10.1016/j.redox.2021.102094

26. Rogers, A.J., Fast, V.G., Sethu, P.: Biomimetic Cardiac Tissue Model Enables the Adaption of Human Induced Pluripotent Stem Cell Cardiomyocytes to Physiological Hemodynamic Loads. Analytical chemistry 88, 9862-9868, doi:10.1021/acs.analchem.6b03105 (2016)

27. Rogers, A.J., et al.: Hemodynamic Stimulation Using the Biomimetic Cardiac Tissue Model (BCTM) Enhances Maturation of Human Induced Pluripotent Stem Cell-Derived Cardiomyocytes. Cells, tissues, organs, 1-13, doi:10.1159/000496934 (2019)

28. Rogers, A.J., Miller, J.M., Kannappan, R., Sethu, P.: Cardiac Tissue Chips (CTCs) for Modelling Cardiovascular Disease. IEEE Trans. Biomed. Eng., 1-1, doi:10.1109/TBME.2019.2905763 (2019)

29. de Tombe, P.P., ter Keurs, H.E.: The velocity of cardiac sarcomere shortening: mechanisms and implications. J. Muscle Res. Cell. Motil. 33, 431-437 (2012). doi:10.1007/s10974-012-9310-0

30. Abdeltawab, H., Hammouda, F.K.K., Miller, J.M.: and et al. Artificial Intelligence Based Framework to Quantify the Cardiomyocyte Structural Integrity in Heart Slices. Cardiovasc. Eng. Technol., doi:10.1007/s13239-021-00571-6 (2021)

31. Parikh, S.S., et al.: Thyroid and Glucocorticoid Hormones Promote Functional T-Tubule Development in Human-Induced Pluripotent Stem Cell-Derived Cardiomyocytes. Circ. Res. 121, 1323-1330 (2017). doi:10.1161/CIRCRESAHA.117.311920

32. Yang, X., et al.: Tri-iodo-l-thyronine promotes the maturation of human cardiomyocytes-derived from induced pluripotent stem cells. J. Mol. Cell. Cardiol. 72, 296-304 (2014). doi:10.1016/j.yjmcc.2014.04.005

33. Barnes, P.J.: Anti-inflammatory actions of glucocoticoids: molecular mechanisms. Chinical Sci. 94, 557-572 (1998). doi:10.1042/cs0940557

34. Oakley, R.H., Cidlowski, J.A.: Glucocorticoid signaling in the heart: A cardiomyocyte perspective. J. Steroid Biochem. Mol. Biol. 153, 27-34 (2015). doi:10.1016/j.jsbmb.2015.03.009

35. Mohamed, T.M., et al.: Chemical Enhancement of In Vitro and In Vivo Direct Cardiac Reprogramming. Circulation 135, 978-995, doi:10.1161/CIRCULATIONAHA.116.024692 (2017)

36. Rukuda, R., El-Sammak, R.M.-J.,H., Beisaw, A.: and et al. Stimulation of glycolysis promotes cardiomyocyte proliferation after injury in adult zebrafish. EMBO Rep. 21, doi:10.15252/embr.201949752 (2020)

37. Scuderi, G.J., Butcher, J.: Naturally Engineered Maturation of Cardiomyocytes. Front. Cell. Dev. Biol. 5, 50 (2017). doi:10.3389/fcell.2017.00050

38. Ruan, J.L., et al.: Mechanical Stress Conditioning and Electrical Stimulation Promote Contractility and Force Maturation of Induced Pluripotent Stem Cell-Derived Human Cardiac Tissue. Circulation. 134, 1557-1567 (2016). doi:10.1161/CIRCULATIONAHA.114.014998

39. Govoni, M., Muscari, C., Guarnieri, C., Giordano, E. Mechanostimulation protocols for cardiac tissue engineering. Biomed Res Int. 2013, 918640, doi:10.1155/2013/918640 (2013) 
40. Mohr-Kahaly, S., a., G.K.: J. M. [Cardiovascular effects of thyroid hormones]. Z. Kardiol 85, 219-231 (1996)

41. Mahdavi, V.: A. P. C. a. B. N.-G. Cardiac alpha- and beta-myosin heavy chain genes are organized in tandem. Proc. Natl. Acad. Sci. U.S.A. 81, 2626-2630 (1984). doi:10.1073/pnas.81.9.2626

42. Xue, Q., Patterson, A.J., Xiao, D., Zhang, L.: Glucocorticoid modulates angiotensin II receptor expression patterns and protects the heart from ischemia and reperfusion injury. PLoS One. 9, e106827 (2014). doi:10.1371/journal.pone.0106827

43. Wester, M., et al.: Glucocorticoid stimulation increases cardiac contractility by SGK1-dependent SOCE-activation in rat cardiac myocytes. PLoS One. 14, e0222341 (2019). doi:10.1371/journal.pone.0222341

44. Itagaki, K., et al.: Dexamethasone stimulates store-operated calcium entry and protein degradation in cultured L6 myotubes through a phospholipase A(2)-dependent mechanism. Am. J. Physiol. Cell. Physiol. 298, C1127-C1139 (2010). doi:10.1152/ajpcell.00309.2009

\section{Figures}




\section{Figure 1}
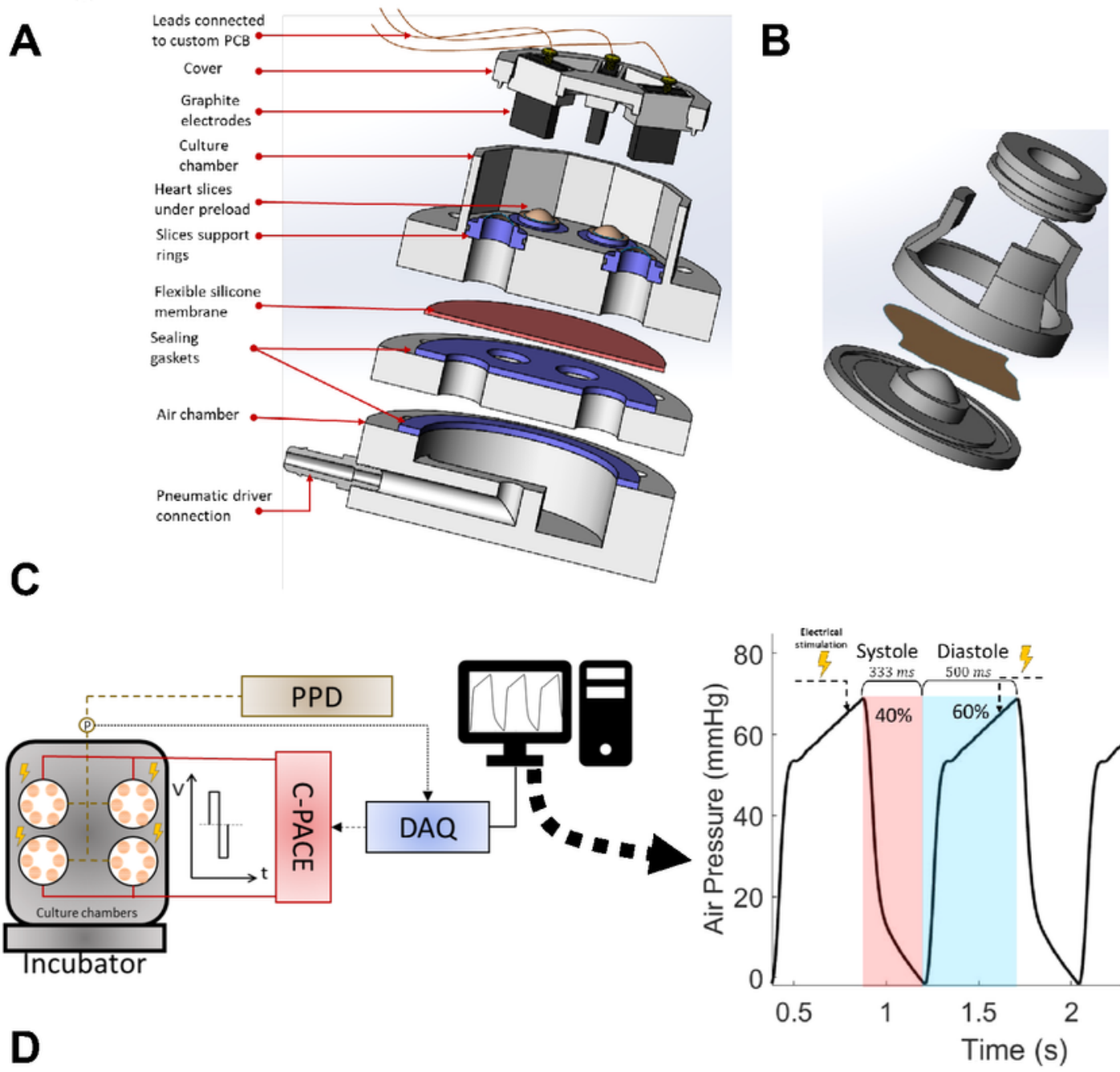

\section{C}
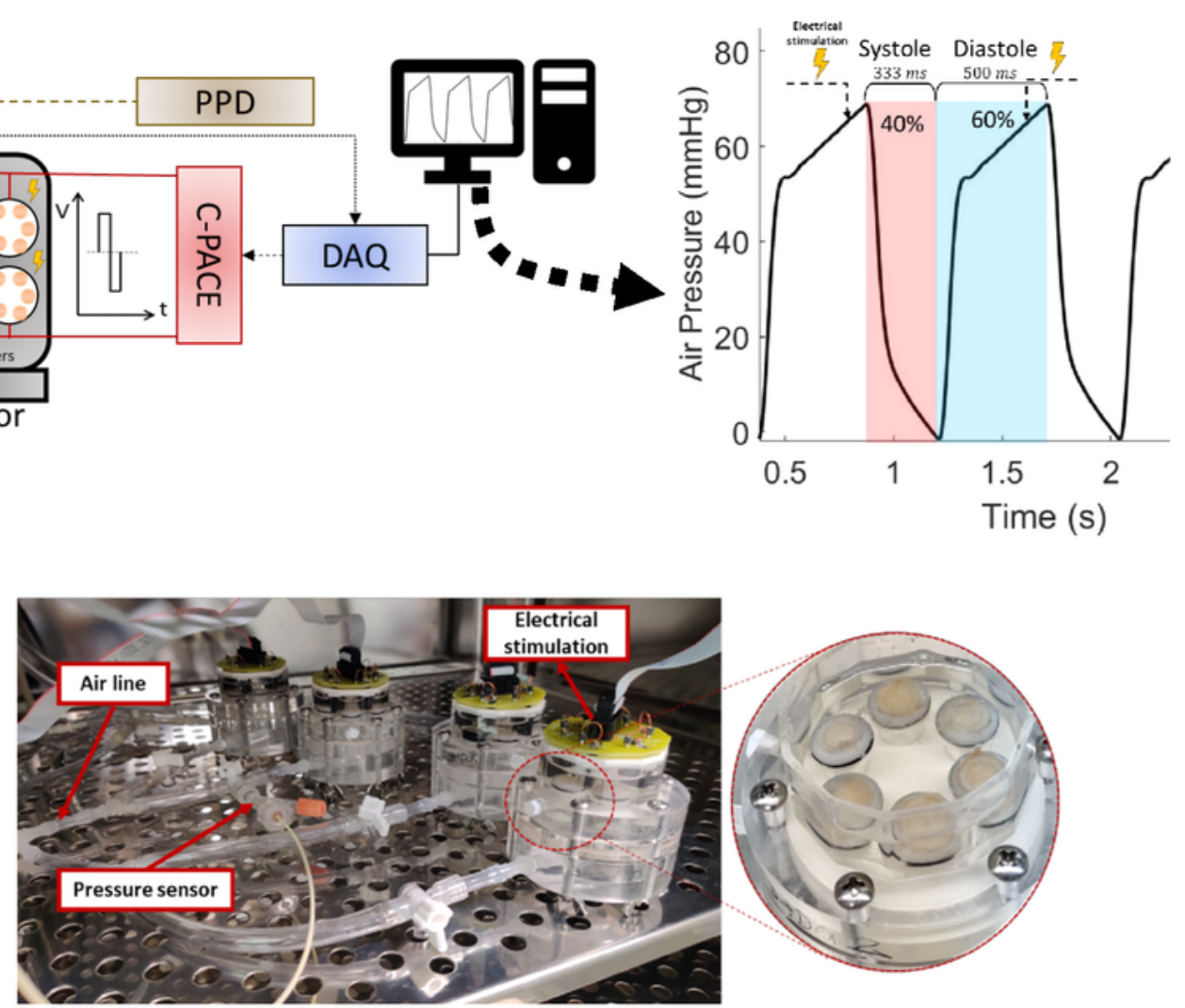

\section{Figure 1}

Cardiac tissue culture model (CTCM). (A) Schematic illustration of the CTCM. The tissue slices are attached to 7-mm diameter support rings shown in blue within the culture chamber of the device. The culture chamber was separated from the air chamber by a thin flexible silicone membrane. Between each chamber, a sealing gasket was placed to prevent leaks. The cover for the device contains graphite electrodes that provide electrical stimulation. (B) A diagram depicting the timing of the electrical 
stimulation in relation to the pressure within the air chamber controlled by the programmable pneumatic driver (PPD). Using a pressure probe sensor, a data acquisition device (DAQ) was utilized. When the pressure within the culture chamber reaches a specified threshold, an impulse signal is sent to the CPACE-EM device to induce electrical stimulation. (C) Image of four CTCM devices set up on a shelf of an incubator. These four devices are connected to a single PPD by air lines and a pressure sensor is inserted into a hemostatic valve to monitor the pressure within the air line. Each device can accommodate 6 tissue slices. 


\section{Figure 2}

A
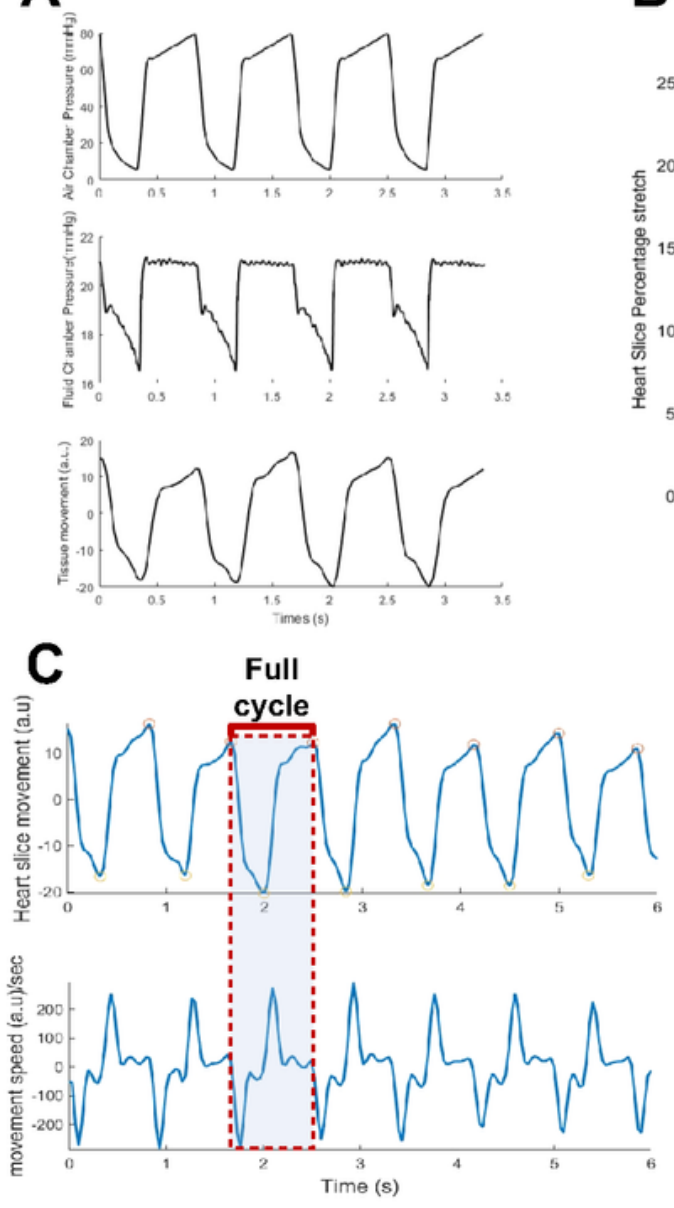

E Cycle Time

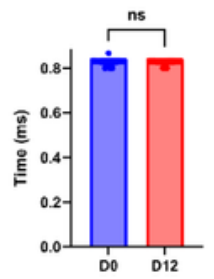

Tissue movement amplitude

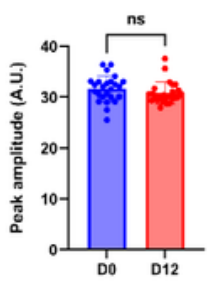

B

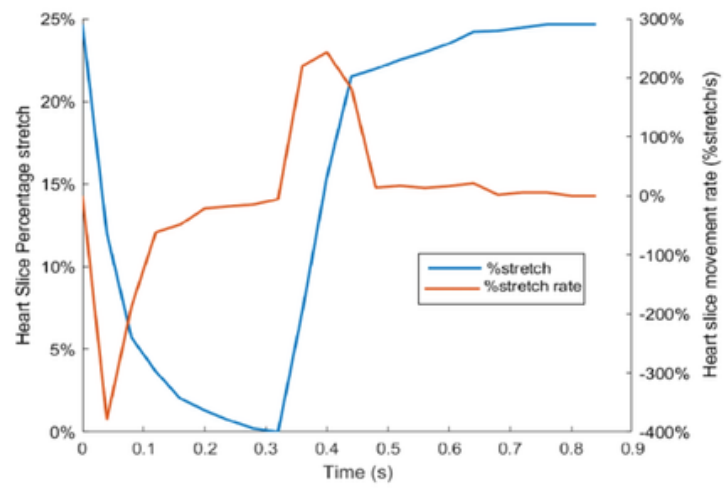

D
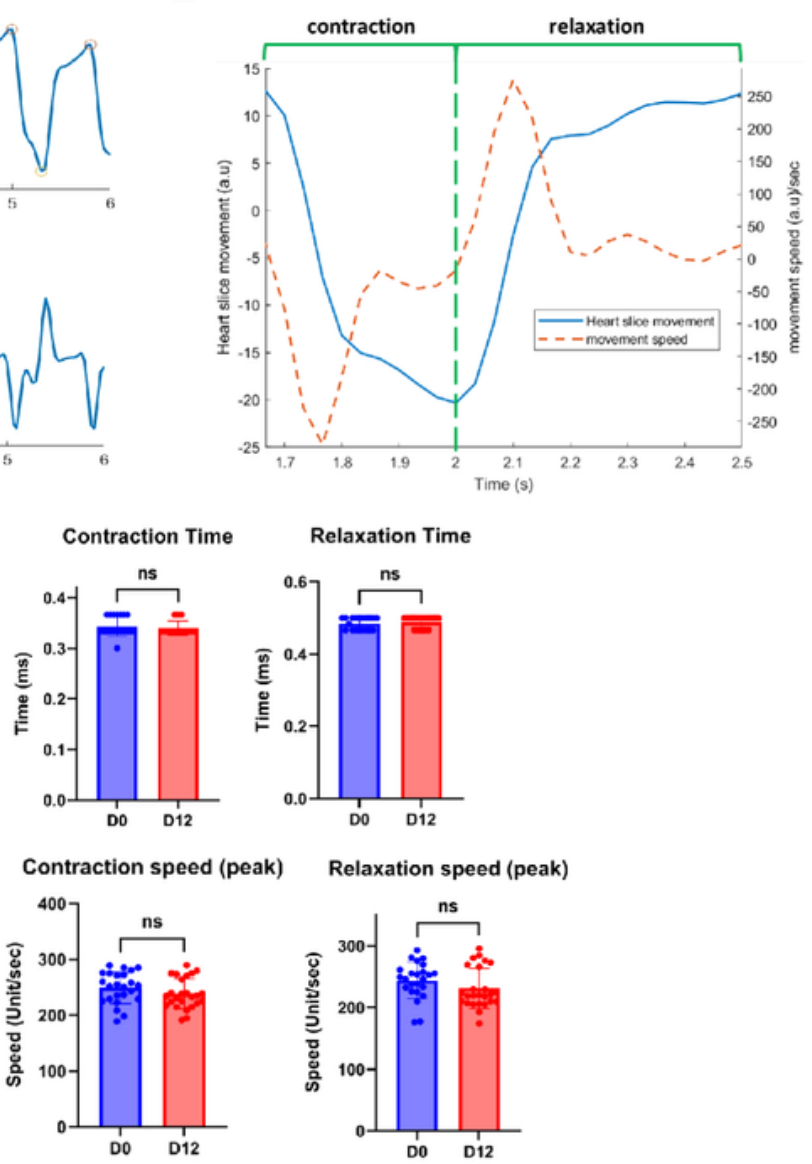

Relaxation speed (peak)

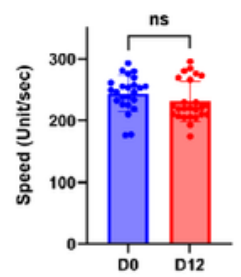

Figure 2

Characterization of the CTCM and evaluation of the heart slice stretches over time in culture. (A) representative trances of the air chamber pressure (top), fluid chamber pressure (middle), and tissue movement (bottom) measurements verified that the air chamber pressure changes the fluid chamber pressure, which induces a corresponding tissue slice movement. (B) representative traces of the percent stretch (blue) of the tissue slices which corresponds with the percent stretch rate (orange). (C) The heart 
slice movement (top) measured was consistent with the measured movement speed (bottom). (D) representative trace of the heart slice cycle movements (top) and speed (bottom). (E) Quantification of the cycle time $(n=19)$, contraction time $(n=19)$, relaxation time $(n=19)$, tissue movement $(n=25)$, peak contraction speed $(n=24-25)$, and peak relaxation speeds $(n=24-25)$; all parameters remained unchanged from 0 to 12 days in culture.

\section{Figure 3}

\section{B}

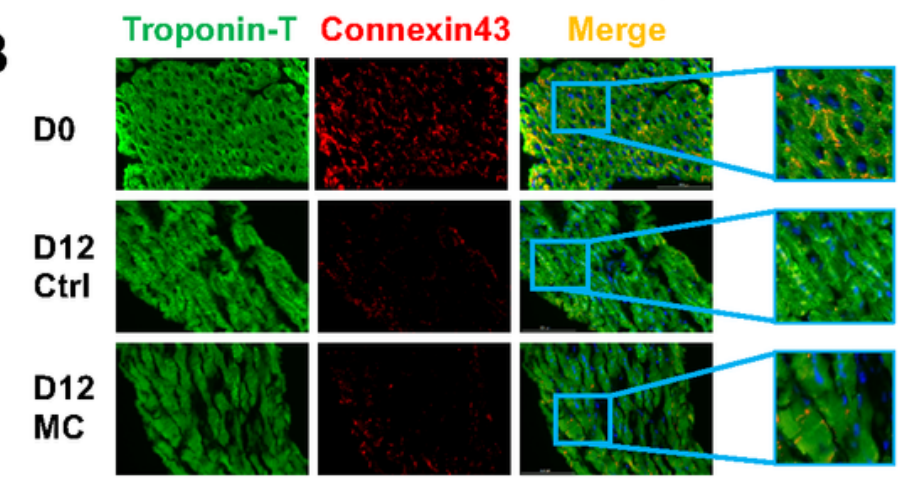

A
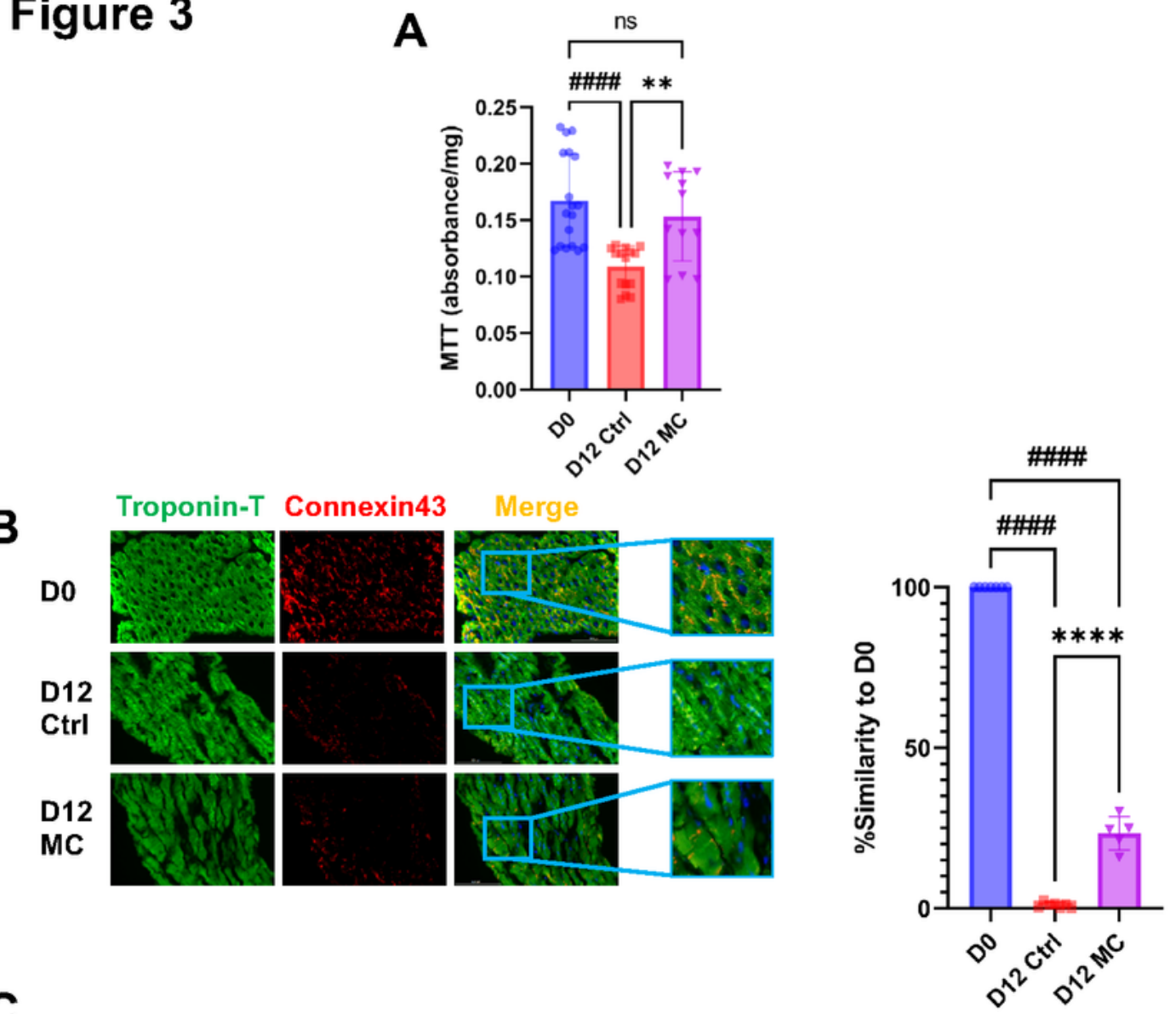

C
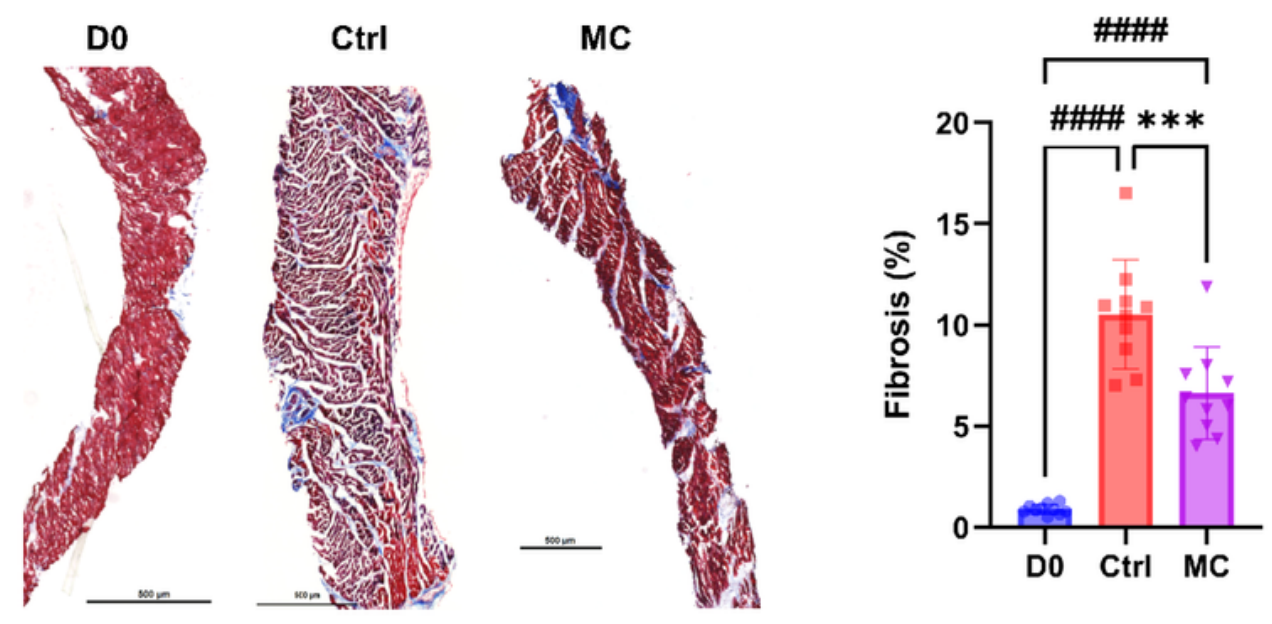

Figure 3 
Application of mechanical stimulation partially improves tissue slice viability and structural integrity after

12 days in culture. (A) Bar graph shows quantification of the MTT viability of fresh heart slices (D0) or heart slices culture for 12 days either in static culture (D12 Ctrl) or in CTCM (D12 MC) (n=12-18, \#\#\#\#p<0.0001 compared to D0 and **p<0.01 compared to D12 Ctrl). (B) Representative immunofluorescence images for troponin-T (green), connexin 43 (red), and DAPI (blue) for freshly isolated heart slices (D0) or heart slices cultured for 12 days under static conditions (Ctrl) or CTCM conditions (MC) Artificial intelligence quantification of the heart tissue structural integrity ( $n=5-7$ slices

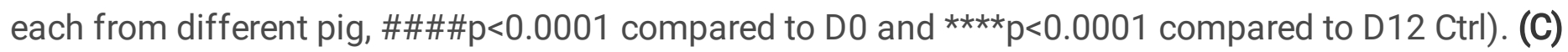
Representative images (left) and quantification (right) for heart slices stained with Masson's trichrome

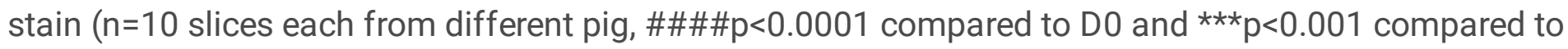
D12 Ctrl). 


\section{Figure 4}

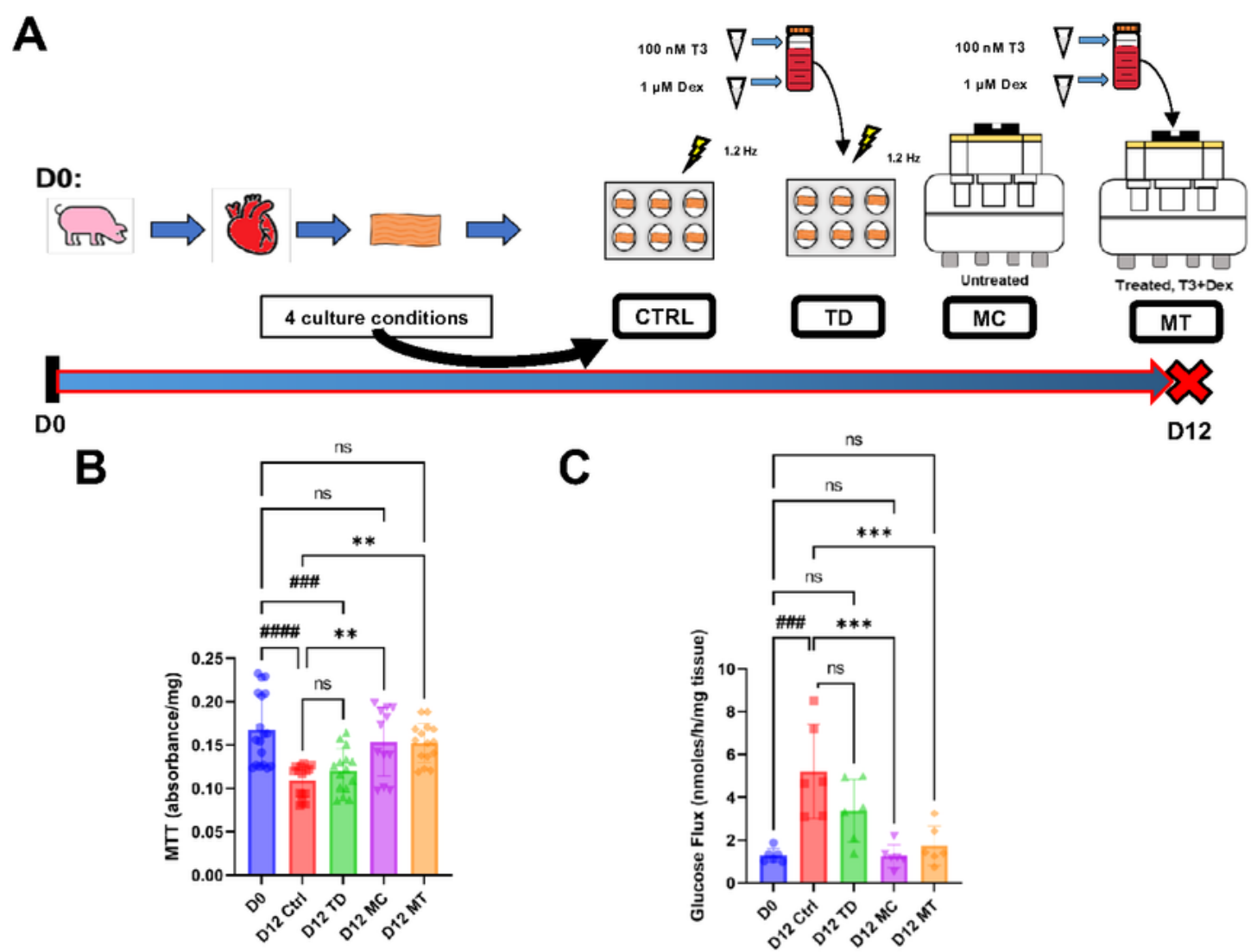

D

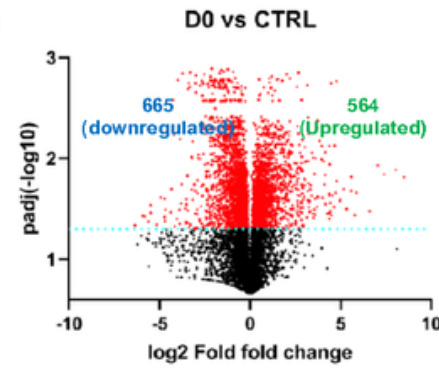

D0 vs MT

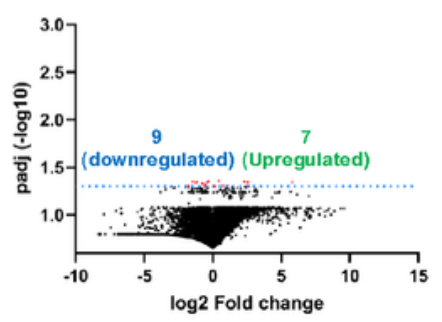

$\mathbf{E}$

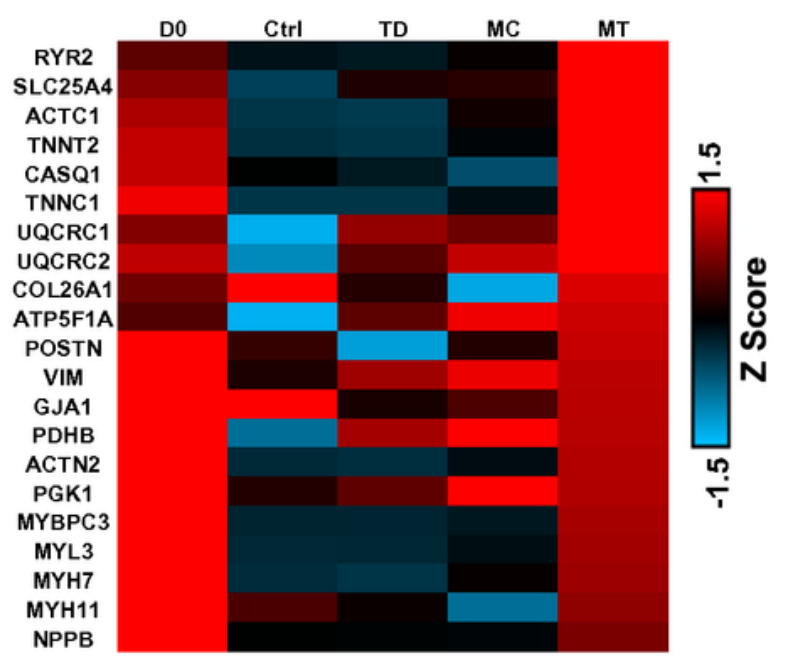

Figure 4

Combination of mechanical and humoral stimulation results in maintained viability, metabolic activity, and transcriptional profile in heart slices for 12 days. (A) Schematic representation of the experimental design depicting the four culture conditions that were used to assess the effect of combining mechanical stimulation and T3/Dex addition to the culture media over a course of 12 days. (B) Bar graph shows quantification of viability 12 days post culture in all 4 culture conditions (Ctrl, TD, MC, and MT) compared 
to fresh heart slices (D0) ( $n=12-18$ slices, \#\#\#\#p<0.0001, \#\#\#p<0.001 compared to D0 and ${ }^{* *} p<0.01$ compared to D12 Ctrl). (C) Bar graph shows the quantification of glucose flux 12 days post culture in all 4 culture conditions (Ctrl, TD, MC, and MT) compared to fresh heart slices (D0) ( $n=6$ slices, $\# \# \# p<0.001$, compared to D0 and ${ }^{* \star *} p<0.001$ compared to D12 Ctrl). (D) Volcano plots showing the differentially expressed genes in fresh heart slices (D0) compared to heart slices cultured under static conditions (Ctrl) (top) or MT conditions (MT) (bottom) for 10-12 days $(n=2-4)(E)$ Heatmap for the major cardiac gene for heart slices cultured under each culture condition $(n=2-4)$. 


\section{Figure 5}

A
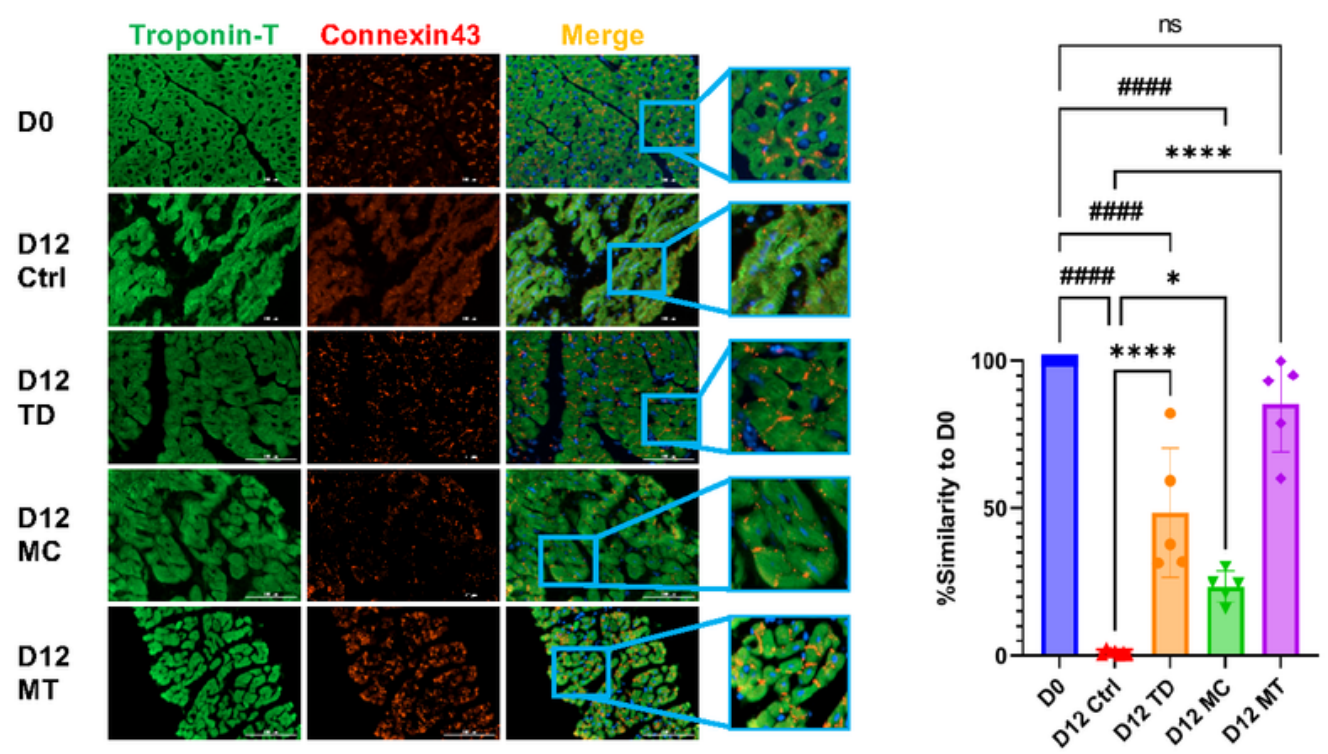

B
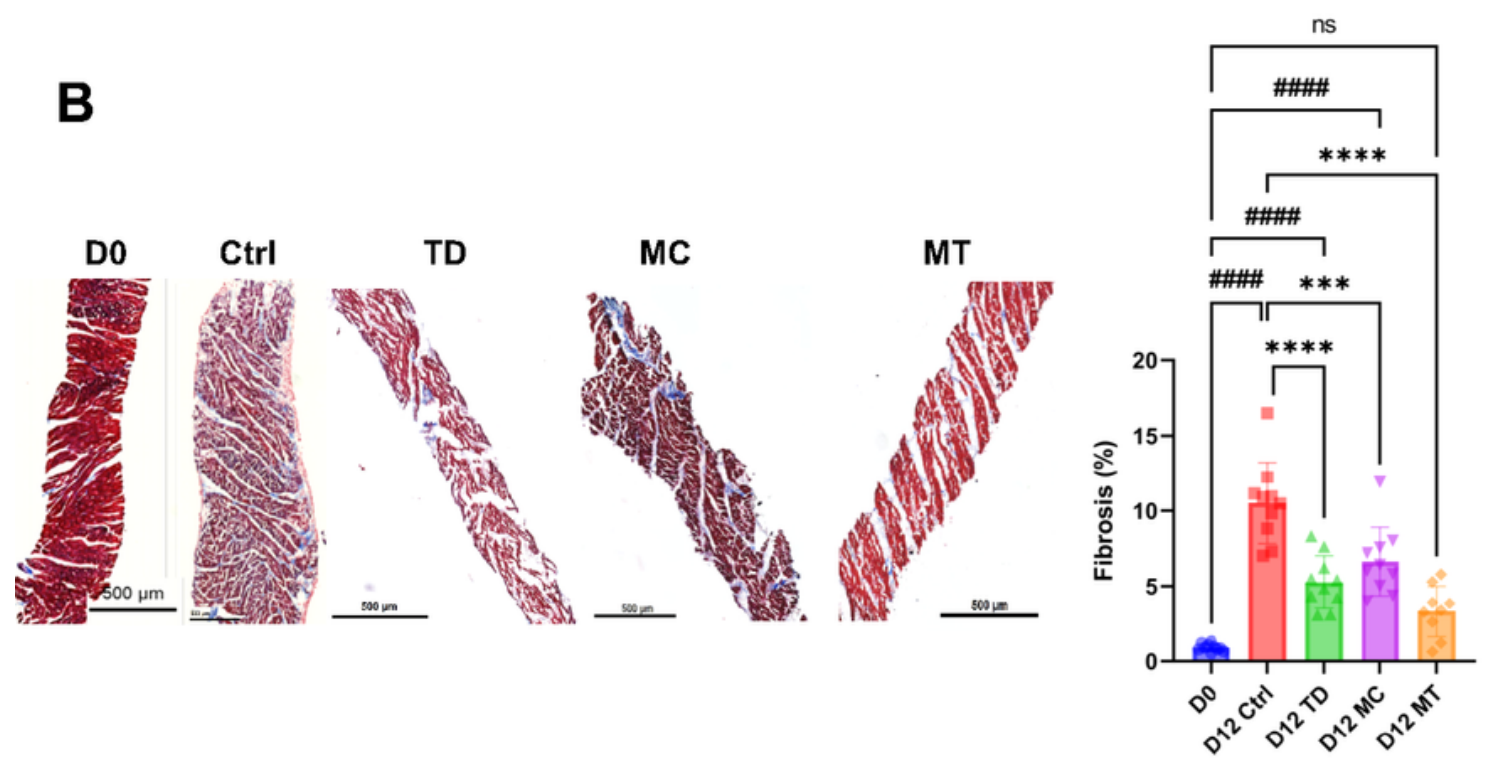

Figure 5

Cardiac tissue structure in slices cultured for 12 days under MT conditions is similar to fresh heart slices. (A) Representative immunofluorescence images for troponin-T (green), connexin 43 (red), and DAPI (blue) for freshly isolated heart slices (D0) or heart slices cultured for 12 days under all 4 culture conditions. Artificial intelligence quantification of the heart tissue structural integrity $(n=5-7$ slices, \#\#\#\#p<0.0001 compared to D0 and * $p<0.05$, or ${ }^{* * *} p<0.0001$ compared to D12 Ctrl). (B) Representative 
images (left) and quantification (right) for heart slices stained with Masson's trichrome stain ( $\mathrm{n}=9-10$

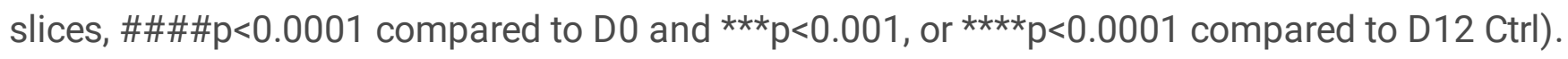

\section{Figure 6}

A
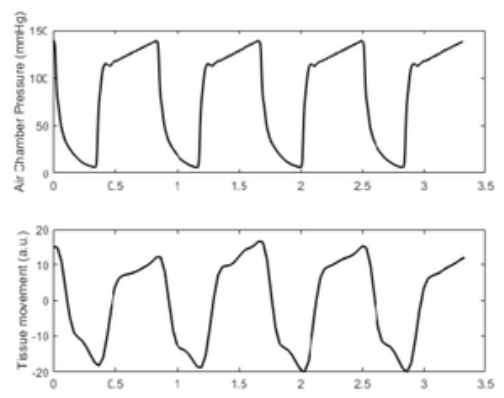

B
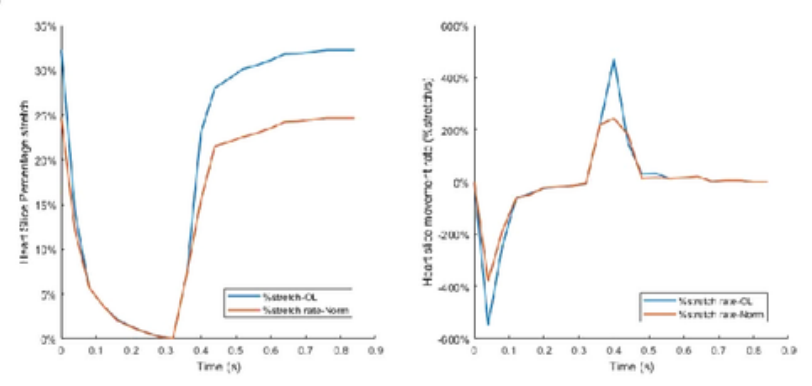

C

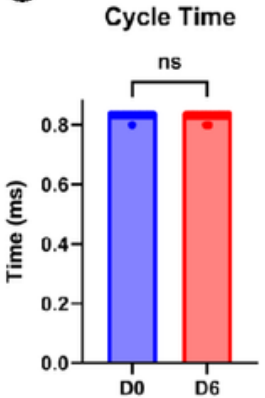

Contraction Time

Relaxation Time
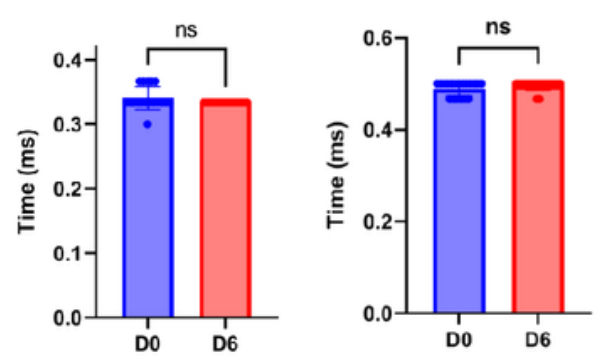

Tissue movement amplitude

Contraction speed (peak)

Relaxation speed (peak)
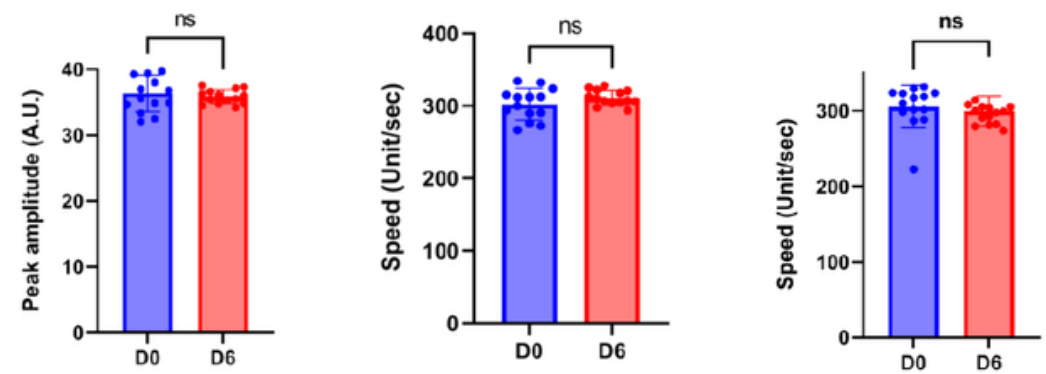

Figure 6 
Induction of pathological overload in heart slices cultured in CTCM device. (A) representative trances of the air chamber pressure (top), and tissue movement (bottom) measurements verified synchronization between the air chamber pressure and the corresponding tissue movement. (B) representative traces of the percent stretch (left) and stretch rate (right) of normal load (orange) and overload (blue) tissue slices (C) Bar graphs showing quantification of the cycle time $(n=19)$, contraction time $(n=18-19)$, relaxation time $(n=19)$, tissue movement amplitude $(n=14)$, peak contraction speed $(n=14)$, and peak relaxation speeds $(n=14-15)$ demonstrates that these parameters remained consistent over 6 days of overload culture. 


\section{Figure 7}

A

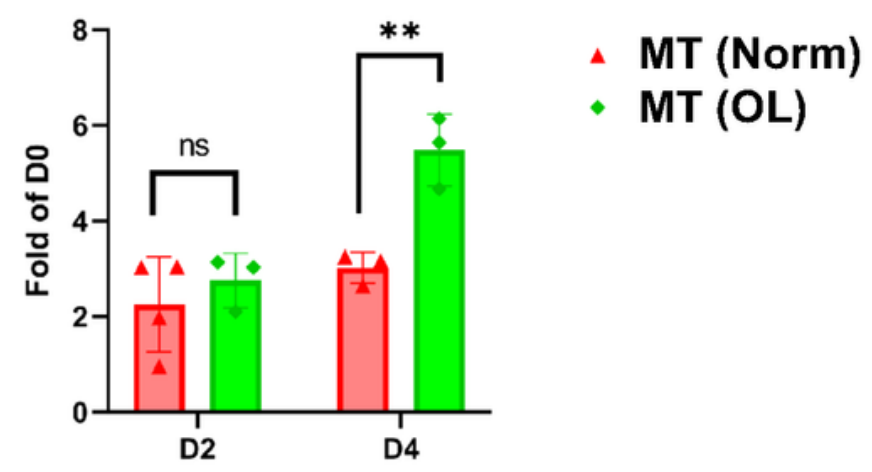

B
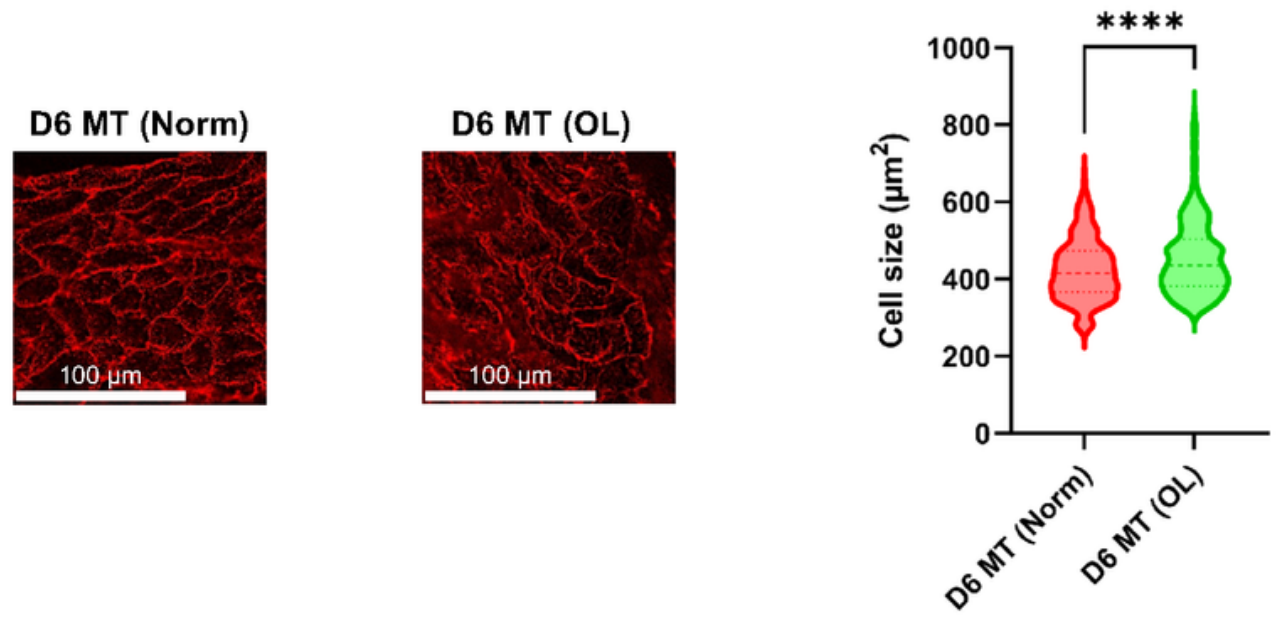

Figure 7

Overload in CTCM induces pathological hypertrophy. (A) Bar graph quantification of NT-Pro-BNP concentration in culture media from heart slices cultured under MT normal load (Norm) or overload (OL) conditions ( $n=3-4,{ }^{* \star} p<0.01$, compared to normal load) (B) Representative images for heart slices stained with WGA (left) and cell size quantification (right) ( $n=330-369$ cells; ${ }^{* \star \star *} \mathrm{p}<0.0001$ compared to normal load). 


\section{Supplementary Files}

This is a list of supplementary files associated with this preprint. Click to download.

- SupplementalFigures.pdf

- Supplementarycode.pdf

- Supplementarysourcedata.xlsx

- Supplementarymovie1.mov 